International Journal of Distributed and Parallel Systems (IJDPS) Vol.2, No.6, November 2011

\title{
TAILORING OF FLATTENED DISPERSION IN TRIANGULAR-LATTICE PHOTONIC CRYSTAL FIBER
}

\author{
${ }^{1}$ Sandhir Kumar Singh, ${ }^{2}$ D. K. Singh, ${ }^{3}$ P. Mahto \\ ${ }^{1}$ Department of Engineering Physics, NETGI, Ranchi, Jaharkhand, India \\ Sandhir1975@yahoo.co.in \\ ${ }^{2}$ Department of Electronics and Comm. Engg, BIT Sindri, Dhanbad, Jharkhand \\ dksingh_bit@yahoo.com \\ ${ }^{3}$ P.G. Depatment of Physics, VBU Hazaribag, Jharkhand. \\ pmahtovbu@rediffmail.com
}

\begin{abstract}
:
The interest of researchers and engineers in several laboratories, since the 1980s, has been attracted by the ability to structure materials on the scale of the optical wavelength, a fraction of micrometers or less, in order to develop new optical medium, known as photonic crystals. Photonic crystals rely on a regular morphological microstructure of air-holes, incorporated into the material, which radically alters its optical properties. In Photonic Crystal Fiber $(P C F)$ it is possible to realize flat dispersion over a wide wavelength range that cannot be realized with a conventional singlemode fiber. In PCFs, the dispersion can be controlled and tailored with unprecedented freedom. In fact, due to the high refractive index difference between silica and air, and to the flexibility of changing airhole sizes and patterns, the waveguide contribution to the dispersion parameter can be significantly changed, thus obtaining unusual position of the zero dispersion wavelength, as well as particular values of the dispersion curve slope. In particular, by manipulating the air-hole radius or the lattice period of the micro structured cladding, it is possible to control the zero-dispersion wavelength, which can be tuned over a very wide range, or the dispersion curves, which can be engineered to be ultra flattened. In this paper the geometric parameters of triangular PCF have been properly changed to optimize the dispersion compensation over a wide wavelength range.
\end{abstract}

Keywords: Photonic crystal fiber (PCFs), Dispersion compensating fiber (DCFs), Single mode fiber (SMFs), Wavelength division multiplexing (WDM), Dispersion (D)

\section{Introduction}

Due to the flexibility for the cross section design, photonic crystal fibers (PCFs) have achieved excellent properties in dispersion[1-10], single polarization single mode[11-13], nonlinearity[14], and effective mode area[15-17], and also excellent performances in the applications of fiber sensors[18-19], fiber lasers[20-22] and nonlinear optics[23-26] over the past several years. Large numbers of research papers have highlighted some optical properties of the PCFs such as ultrahigh birefringence and unique chromatic dispersion, which are almost impossible for the conventional optical fibers.

The tendency for different light wavelengths to travel at different speeds is a crucial factor in the telecommunication system design. A sequence of short light pulses carries the digitized information. Each of these is formed from a spread of wavelengths and, as a result of chromatic dispersion, it broadens as it travels, thus obscuring the signal. The magnitude of the dispersion 
changes with the wavelength, passing through zero at $1.3 \mu \mathrm{m}$ in conventional optical fibers. For example, as the air-holes get larger, the PCF core becomes more and more isolated, until it resembles an isolated strand of silica glass suspended by six thin webs of glass. If the whole structure is made very small, the zero-dispersion wavelength can be shifted to the visible light, since the group velocity dispersion is radically affected by pure waveguide dispersion. On the contrary, very flat dispersion curves can be obtained in certain wavelength ranges in PCFs with small air-holes, that is with low air-filling fraction. First of all, the study of the dispersion properties of triangular PCFs with a high air-filling fraction, that is with small hole-to-hole spacing and large air-holes, is designed to compensate the anomalous dispersion and the dispersion slope of single-mode fibers [27-29]. In particular, the geometric parameters which characterize these triangular PCFs have been chosen to optimize the fiber length and the dispersion compensation over a wide wavelength range.

\section{Dispersion compensation in triangular lattice PCFs}

PCFs with a high air-filling fraction have been designed in order to compensate the anomalous dispersion and the dispersion slope of SMFs. In fact, their chromatic dispersion limits the data transmission rate in broadband wavelength division multiplexing (WDM) systems. In particular, it becomes a critical issue as soon as the transmission bit-rate increases over $10 \mathrm{~Gb} / \mathrm{s}$. The positive dispersion of installed fibers can be compensated by dispersion compensating fibers (DCFs) with a large dispersion of opposite sign. For WDM systems this goal must be achieved over a broad wavelength range around $1550 \mathrm{~nm}$, thus implying, besides large negative dispersion values, a proper negative dispersion slope. The present analysis has demonstrated that PCFs can be exploited to this aim. In fact, their dispersion properties can be modified with high flexibility, since the large refractive index variation between silica and air permits to achieve significant waveguide dispersion over a wide wavelength range. PCFs with large air-holes have been already proposed for dispersion compensation, even though their description has been performed through a simplified model consisting of a silica core in air [30]. When the wavelength increases, this approximation gets worse.

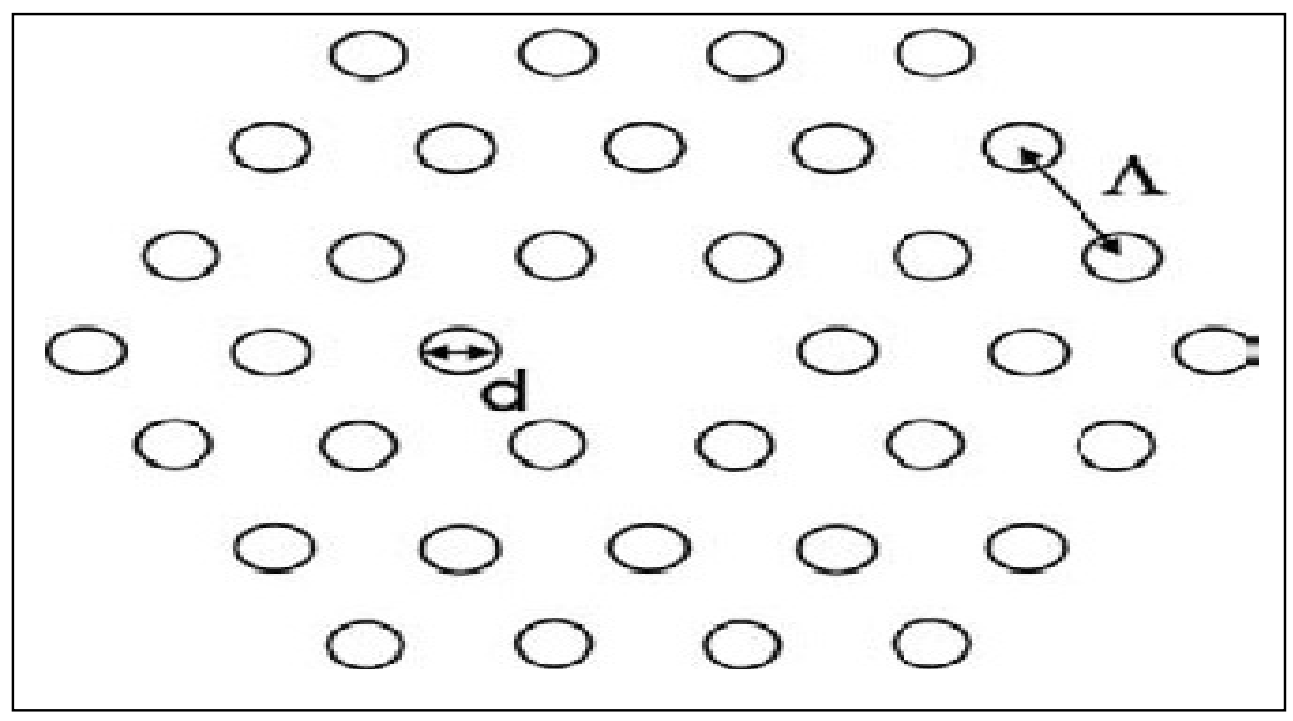

Fig. 1: Cross-section of a triangular PCF with the air-hole diameter $d$ and the pitch $\Lambda$. 
International Journal of Distributed and Parallel Systems (IJDPS) Vol.2, No.6, November 2011

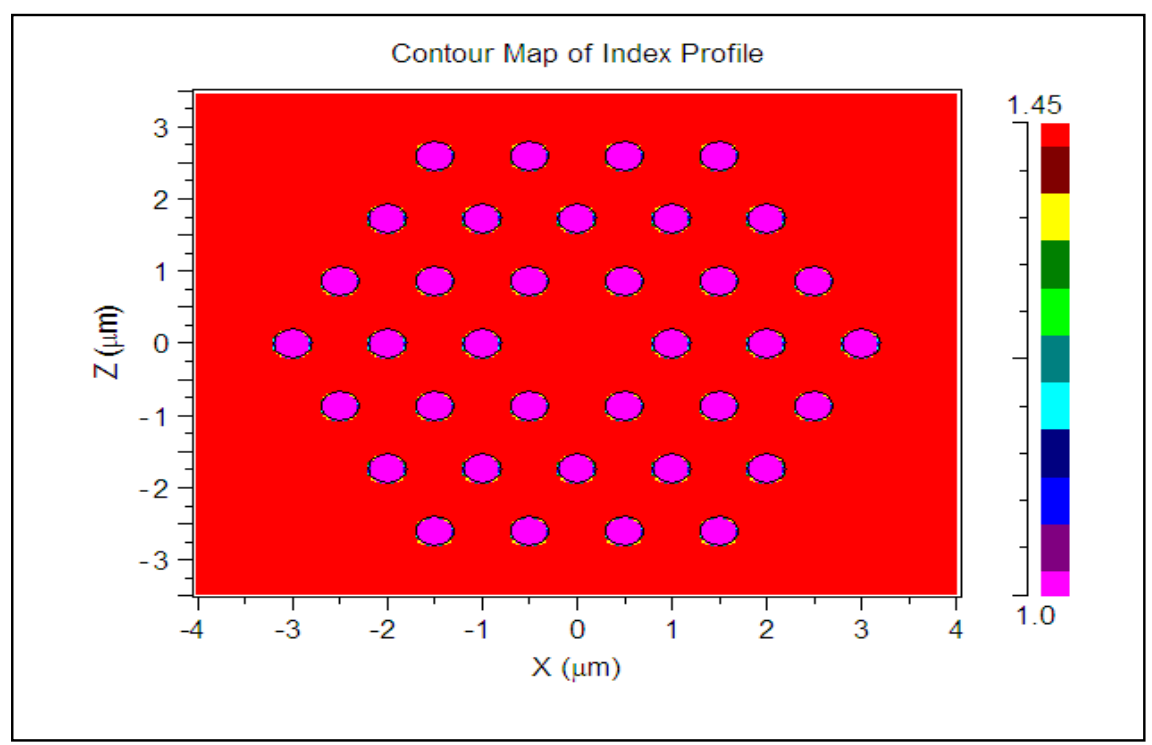

Fig. 2 : Index profile of the triangular lattice PCF structure
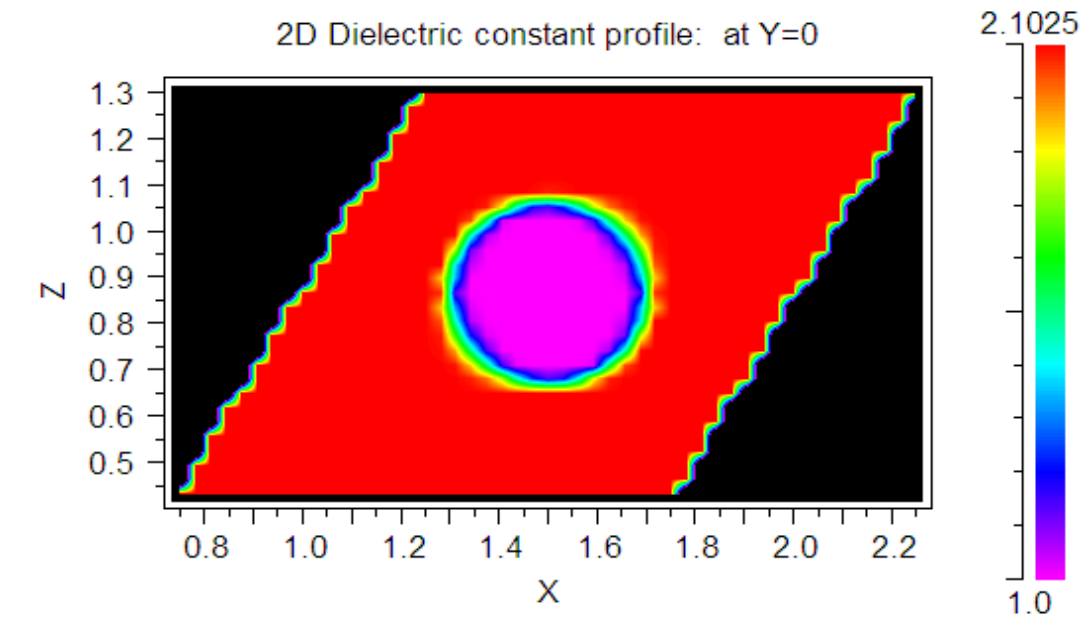

Fig.3 : Mode view of the triangular lattice PCF structure 
International Journal of Distributed and Parallel Systems (IJDPS) Vol.2, No.6, November 2011

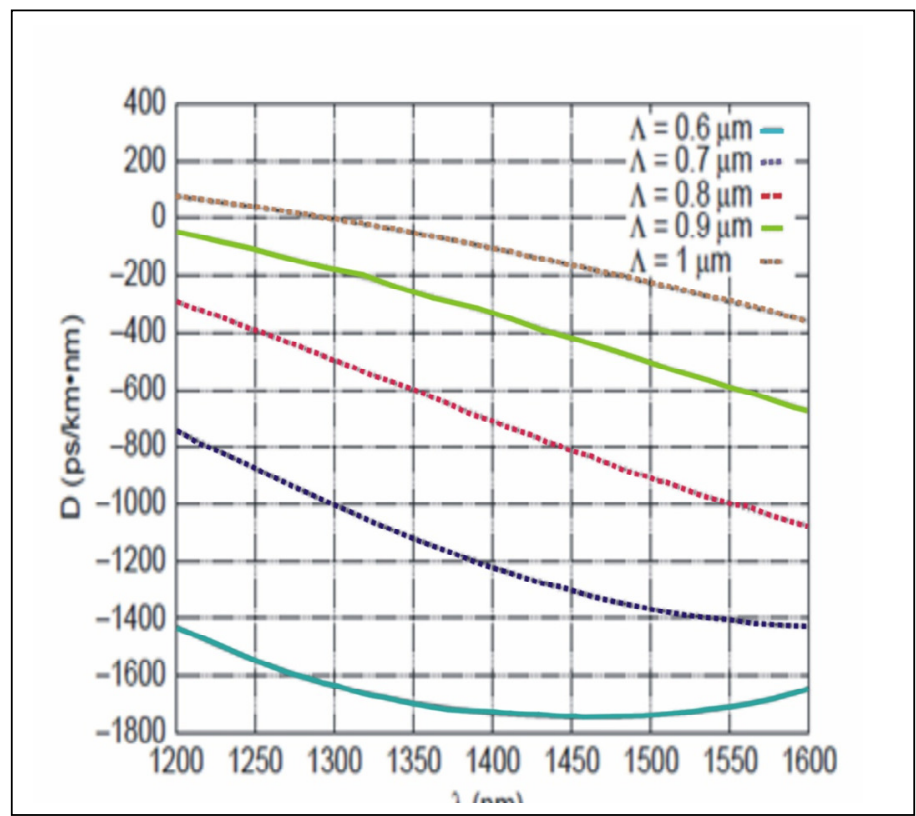

Fig. 4: Dispersion parameter for PCFs with $\mathrm{d} / \Lambda=0.9 \mu \mathrm{m}$ and

different $\Lambda$ values

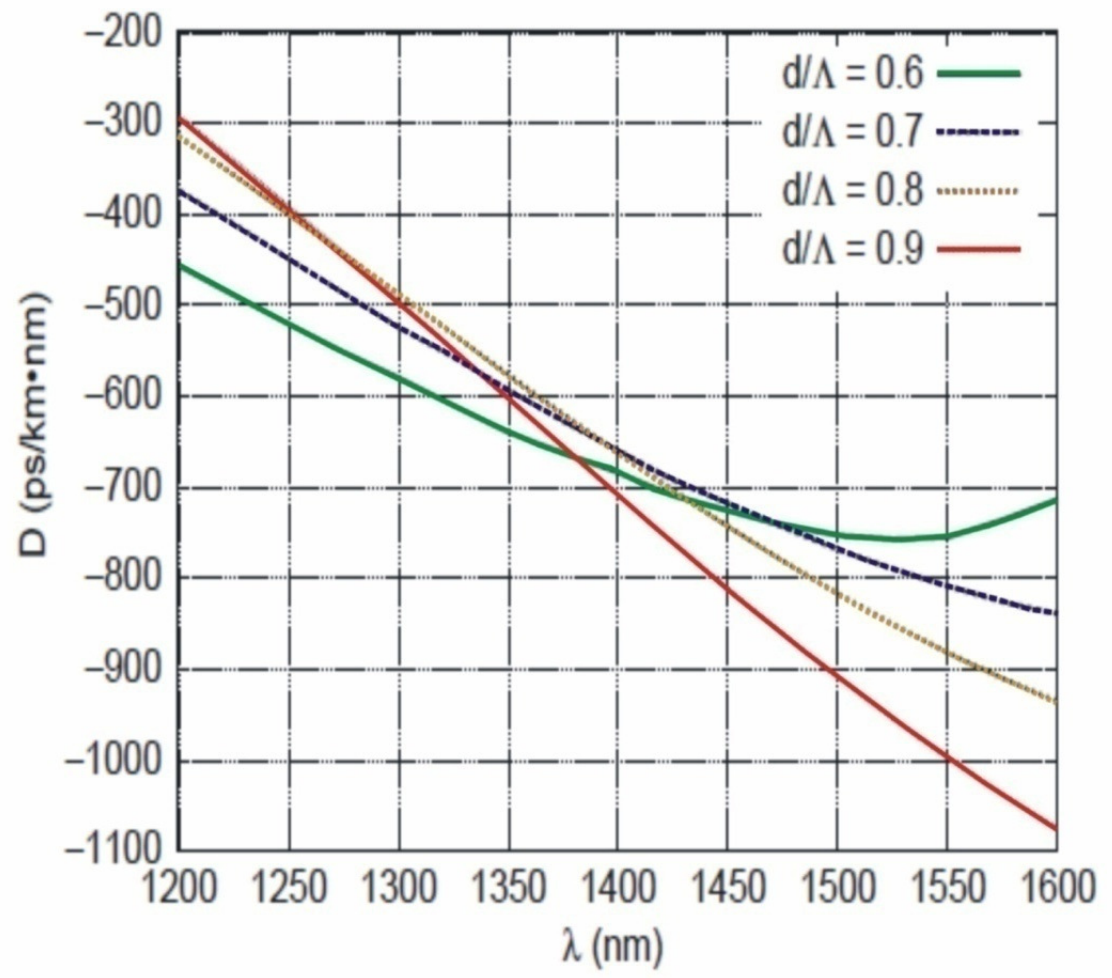

Fig. 5: Dispersion parameter for PCFs with $\Lambda=0.8 \mu \mathrm{m}$ and different $d / \Lambda$ values 


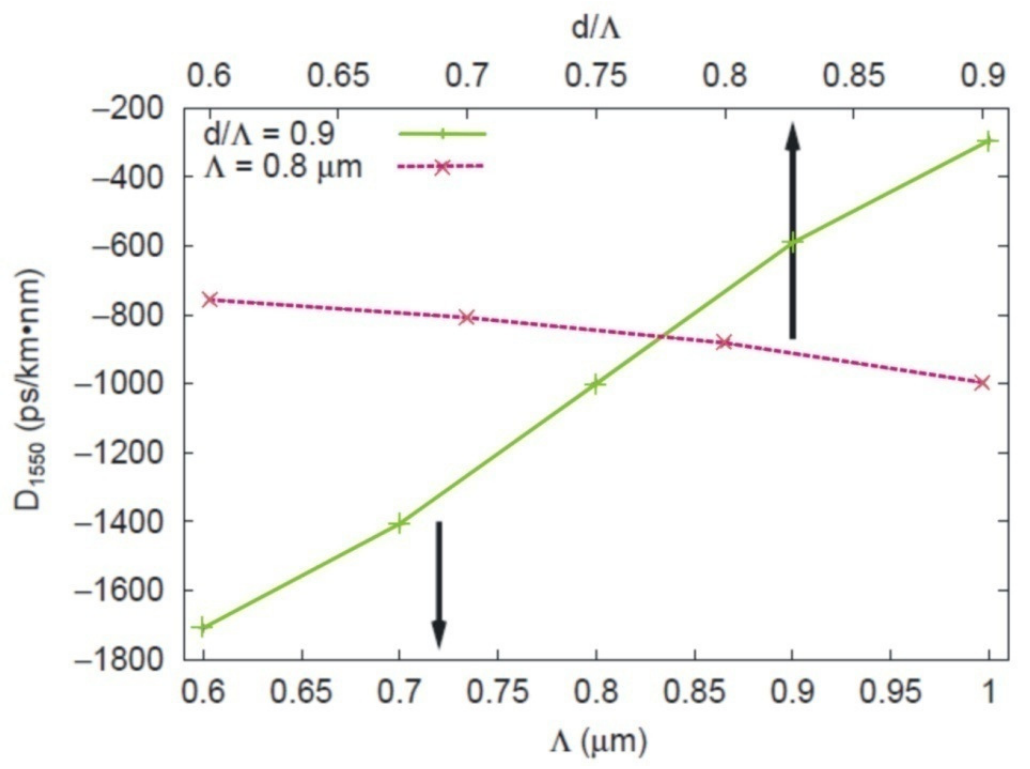

Fig. 6: Chromatic dispersion value at $1550 \mathrm{~nm}$ for the different triangular PCFs considered

\section{Results and Discussion}

In this study, the design of triangular PCFs has been optimized by properly tailoring the air-hole diameter $d$ and the pitch $\Lambda$, as shown in Fig. 1, in order to compensate both the positive dispersion and the positive dispersion slope of single-mode fibers over a wavelength range around $1550 \mathrm{~nm}$. To this aim, triangular PCFs with large air-holes and a small pitch, that is with a small core diameter $d_{\text {core }}=2 \Lambda-d=\Lambda \cdot(2-d / \Lambda)$, have been considered. For the triangular PCFs here studied, a proper number of air-hole rings has been considered in order for the solution to converge toward that of a fiber with an infinite photonic crystal cladding. This results in a considerable reduction of the leakage losses [31]. In this paper the radiation field is evaluated by using FEM formulation. It has been shown that, by choosing the ring number between three and ten, leakage losses of fibers with $d / \Lambda$ in the range $0.6-0.9$ can be reduced under the Rayleigh scattering limit $[32,33]$. The dispersion parameter $D$ has been derived in the wavelength range $1200 \mathrm{~nm}-1600 \mathrm{~nm}$. The first fibers considered have $d / \Lambda=0.9$ and $\Lambda$ which varies between 0.6 and $1 \mu \mathrm{m}$. Fig. 4 shows their dispersion parameter $D$ for the wavelengths between 1200 and $1600 \mathrm{~nm}$. $D$ is always negative if $\Lambda<1 \mu \mathrm{m}$ and becomes positive only for the triangular PCF with $\Lambda=1 \mu \mathrm{m}$ when $\lambda<1300 \mathrm{~nm}$. The absolute value of the dispersion parameter increases reducing the hole to hole spacing $\Lambda$. For the triangular PCF with $\Lambda=0.6 \mu \mathrm{m}$ $D$ reaches a value around $-1700 \mathrm{ps} / \mathrm{km} \mathrm{nm}$ at $1550 \mathrm{~nm}$, while for conventional DCFs it is typically $-100 \mathrm{ps} / \mathrm{km} \cdot \mathrm{nm}$ at this wavelength $[30,36]$. The dispersion slope is always negative in the wavelength range considered if $\Lambda \geq 0.7 \mu \mathrm{m}$, while for the PCF with the smallest pitch, $\Lambda=$ $0.6 \mu \mathrm{m}, D$ reaches a minimum at $1475 \mathrm{~nm}$ and then the dispersion slope becomes positive. In order to optimize the PCF design, the effect of $d$ variation has been investigated. For this reason the pitch has been fixed to $\Lambda=0.8 \mu \mathrm{m}$, that is, a middle value between those previously considered, and the ratio $d / \Lambda$ has been varied from 0.9 to 0.6. As shown in Fig. 5, $D$ is always negative in the wavelength range chosen for all the $d / \Lambda$ values. As $d / \Lambda$ decreases from the initial value of 0.9 , the dispersion slope changes and becomes positive for the PCF with $d / \Lambda=0.6$ if $\lambda$ $>1525 \mathrm{~nm}$. The minimum value of $D$ at $1550 \mathrm{~nm}$, around $-1000 \mathrm{ps} / \mathrm{km} / \mathrm{nm}$, has been obtained with the largest air-holes, that is, with $d / \Lambda=0.9$. Results are summarized in Fig. 6, which shows the dispersion parameter values at $1550 \mathrm{~nm}$. Notice that the dispersion value increases 
significantly with $\Lambda$ when $d / \Lambda$ is fixed to 0.9 , while it slowly decreases when the air-holes become larger, as in the case $\Lambda=0.8 \mu \mathrm{m}$. This result suggests important technological considerations. In fact, proper pitch values, rather than high air-filling fractions, allow to get fibers with dispersion values slightly affected by small variations of the air-hole diameter, eventually introduced by the fabrication process.

The anomalous dispersion of an SMF at $1550 \mathrm{~nm}$ is completely compensated by a DCF if

$$
D_{\mathrm{SMF}} \cdot L_{\mathrm{SMF}}+D_{\mathrm{DCF}} \cdot L_{\mathrm{DCF}}=0
$$

where $D_{\mathrm{SMF}}, D_{\mathrm{DCF}}, L_{\mathrm{SMF}}$, and $L_{\mathrm{DCF}}$ are, respectively, the dispersion parameters and the lengths of the single-mode and the dispersion-compensating fibers. For a given SMF, if the absolute value of $D_{\mathrm{DCF}}$ is bigger, the length of the DCF can be shorter. The triangular PCF with $\Lambda=0.6 \mu \mathrm{m}$ and $d / \Lambda=0.9$, which has the largest value of negative dispersion at $1550 \mathrm{~nm}$, as shown in Fig.4, can be about 17 times shorter than a classical DCF. Unfortunately

this fiber has a positive dispersion curve slope in the third window. In fact, the dispersion slope is very important, being the parameter which characterizes the dispersion compensation over a wavelength range. In an SMF the slope of $D(\lambda)$ at $1550 \mathrm{~nm}$ is positive. The two PCFs, with $\Lambda=$ $0.6 \mu \mathrm{m}$ and $d / \Lambda=0.9$ in Fig. 4 and with $\Lambda=0.8 \mu \mathrm{m}$ and $d / \Lambda=0.6$ in Fig. 5, have a positive dispersion slope too, so they are suitable for dispersion compensation only at one wavelength. In particular, the latter PCF has a lower value of $D$ at $1550 \mathrm{~nm},-755 \mathrm{ps} / \mathrm{km} \mathrm{nm}$. All the other PCFs present a negative dispersion slope at $1550 \mathrm{~nm}$ and can be exploited to compensate the anomalous dispersion of an SMF over a wide wavelength range.

\section{CONCLUSIONS}

The analysis performed above have shown that, by properly changing the geometric characteristics of the air-holes in the PCF cross-section, the waveguide contribution to the dispersion parameter can be significantly changed, thus obtaining unusual positions of the zerodispersion wavelength, as well as particular values of the dispersion curve slope. By manipulating the air-hole radius or the lattice period of the micro structured cladding, it is possible to control the zero-dispersion at wavelength around $1550 \mathrm{~nm}$, which can be tuned over a very wide wavelength range, and the dispersion curves can be engineered to be ultra flattened. The PCFs with these characteristics and with a small effective area, that is a high nonlinear coefficient are suitable for a great number of telecommunication applications, such as wavelength conversion or optical parametric amplification. It is believed that the analysed PCF will have promising future in ultra broadband transmission applications.

\section{ACKNOWLEDGEMENT}

We are thankful to Mr. P.H. Joshi of RSoft for providing us software for simulation purpose and Prof. Rajeshawar Singh, Dean Academic Gateway Institute of Engg. \& Tech. Sonipat, Hariyana for motivation and guidance.

\section{REFERENCES:}

[1] Agrawal, A., N. Kejalakshmy, J. Chen, B. M. A. Rahman, and K. T. V. Grattan, "Golden spiral photonic crystal fiber: Polarization and dispersion properties," Opt. Lett., Vol. 33, 2716-2718,2006

[2] Shen, L. P., W. P. Huang, and S. S. Jian, "Design of photonic crystal fibers for dispersion-related applications," J. Lightwave Technol., Vol. 21, 1644-1651, 2003.

[3] Ferrando, A., E. Silvestre, J. J. Miret, and P. Andres, "Nearly zero ultraflattened dispersion in photonic crystal fibers," Opt. Lett., Vol. 25, 790-792, 2000.

[4] Ferrando, A., E. Silvestre, P. Andres, J. Miret, and M. Andres, "Designing the properties of dispersion-flattened photonic crystal fibers," Opt. Express, Vol. 9, 687-697, 2001. 
International Journal of Distributed and Parallel Systems (IJDPS) Vol.2, No.6, November 2011

[5] Saitoh, K., M. Koshiba, T. Hasegawa, and E. Sasaoka, "Chromatic dispersion control in photonic crystal fibers: Application to ultra- flattened dispersion," Opt. Express, Vol. 11, 843-852, 2003.

[6] Poletti F., V. Finazzi, T. M. Monro, N. G. R. Broderick, V. Tse, and D. J. Richardson, "Inverse design and fabrication tolerances of ultra-flattened dispersion holey fibers," Opt. Express, Vol. 13,3728-3736, 2005.

[7] Gérome, F., J.-L. Auguste, and J.-M. Blondy, "Design of dispersion-compensating fibers based on a dual-concentric-core photonic crystal fiber," Opt. Lett., Vol. 29, 2725-2727, 2004.

[8] Huttunen, A. and P. Törma, "Optimization of dual-core and microstructure fiber geometries for dispersion compensation and large mode area," Opt. Express, Vol. 13, 627-635, 2005.

[9] Varshney, S. K., T. Fujisawa, K. Saitoh, and M. Koshiba, "Design and analysis of a broadband dispersion compensating photonic crystal fiber Raman amplifier operating in S-band," Opt. Express, Vol. 14, 3528-3540, 2006.

[10] Yang, S., Y. Zhang, X. Peng, Y. Lu, S. Xie, J. Li, W. Chen, Z. Jiang, J. Peng, and H. $\mathrm{Li}$, "Theoretical study and experimental fabrication of high negative dispersion photonic crystal fiber with large area mode field," Opt. Express, Vol. 14, 3015-3023, 2006.

[11] Ju, J., W. Jin, and M. S. Demokan, "Design of single-polarization single mode photonics crystal fibers," J. Lightwave Technol., Vol. 24, 825-830, 2001.

[12] Saitoh, K. and M. Koshiba, "Single-polarization single-mode photonic crystal fibers," IEEE Photon. Technol. Lett., Vol. 15,1384-1340, 2003.

[13] Kubota, H., S. Kawanishi, S. Koyanagi, M. Tanaka, and S. Yamaguchi, "Absolutely single polarization photonic crystal fiber," IEEE Photon. Technol. Lett., Vol. 16, 182-184, 2004.

[14] Knight, J. C. and D. V. Skryabin, "Nonlinear waveguide opticsand photonic crystal fibers," Opt. Express, Vol. 15, 15365-15376,2007.

[15] Mortensen, N. A., M. D. Nielsen, J. R. Folkenberg, A. Petersson, and H. R. Simonsen, "Improved large-mode-area endlessly single- mode photonic crystal fibers," Opt. Lett., Vol. 28, 393-395, 2003.

[16] Limpert, J., T. Schreiber, S. Nolte, H. Zellmer, T. Tunnermann, R. Iliew, F. Lederer, J. Broeng, G. Vienne, A. Petersson, and C. Jakobsen, "High-power air-clad large-mode-area photonic crystal fiber laser," Opt. Express, Vol. 11, 818-823, 2003.

[17] Folkenberg, J., M. Nielsen, N. Mortensen, C. Jakobsen, and H. Simonsen, "Polarization maintaining large mode area photonic crystal fiber," Opt. Express, Vol. 12, 956-960, 2004.

[18] Dobb, H., K. Kalli, and D. J. Webb, “Temperature-insensitive long period grating sensors in photonic crystal fibre," Eletron. Lett., Vol. 40, 657-658, 2004.

[19] Dong, X. and H. Y. Tam, "Temperature-insensitive strain sensor with polarizationmaintaining photonic crystal fiber based on Sagnac interferometer," Appl. Phys. Lett., Vol. 90, 151113, 2007.

[20] Wadsworth, W. J., J. C. Knight, W. H. Reewes, P. S. J. Russell, and J. Arriaga, "Yb ${ }^{3+}$-doped photonic crystal fibre laser," Eletron. Lett., Vol. 36, 1452-1253, 2000.

[21] Liu, X., X. Zhou, X. Tang, J. Ng, J. Hao, T. Chai, E. Leong, and C. Lu, "Swithable and tunable multiwavelength erbium-doped fiber laser with fiber Bragg grating and photonic crystal fiber," IEEE Photon. Technol. Lett., Vol. 17, 1626-1628, 2005.

[22] Chen, D., "Stable multi-wavelength erbium-doped fiber laser based on photonic crystal fiber Sagnac loop filter," Laser Phys. Lett., Vol. 4, 437-439, 2007.

[23] Broderick, N. G. R., T. M. Monro, P. J. Bennett, and D. J. Richardson, "Nonlinearity in holey optical fibers: Measure- ment and future opportunities," Opt. Lett., Vol. 24, 1395-1397,1999.

[24] Zhu, Z. and T. G. Brown, "Experimental studies of polarization properties of supercontinua generated in a birefringent photonic crystal fiber," Opt. Express, Vol. 12, 791-796, 2004.

[25.] Zhu, Z. and T. G. Brown, "Polarization properties of supercontinuum spectra generated in birefringent photonic crystal fibers," J. Opt. Soc. Am. B, Vol. 21, 249-257, 2004.

[26.] Dudley, J. M. and J. R. Taylor, "Ten years of nonlinear optics in photonic crystal fibre," 
Nature Photonics, Vol. 3, 85-90, 2009.

[27] F. Poli, A. Cucinotta, M. Fuochi, S. Selleri, and L. Vincetti, "Characterization of microstructured optical fibers for wideband dispersion compensation," Journal of Optical Society of America A, vol. 20, pp.1958-1962, Oct. 2003.

[28] F. Poli, A. Cucinotta, M. Fuochi, S. Selleri, and L. Vincetti, "Dispersion and nonlinear properties of triangular photonic crystal fibers with large air-holes and small pitch," in Proc. European Conference on OpticalCommunication ECOC 2003, Rimini, Italy, Sept. 21-25, 2003.

[29] M. Fuochi, F. Poli, S. Selleri, and A. Cucinotta, "Dispersion and dispersion slope compensation through photonic crystal fibers," in Proc.Progress in Electromagnetics Research Symposium PIERS 2003, Honolulu,Hawaii, USA, Oct. 13-16, 2003.

[30] T. A. Birks, D. Mogilevtsev, J. C. Knight, and P. St. J. Russell, "Dispersion compensation using single-material fibers," IEEE Photonics Technology Letters, vol. 11, pp. 674-676, June 1999.

[31] A. Cucinotta, S. Selleri, L. Vincetti, and M. Zoboli, "Holey fiber analysis through the finite-element method," IEEE Photonics Technology Letters, vol. 14, pp. 1530-1532, Nov. 2002.

[32] T. M. Monro, D. J. Richardson, N. G. R. Broderick, and P. J. Bennett, "Modeling large air fraction holey optical fibers," IEEE/OSA Journal of Lightwave Technology, vol. 18, pp. 50-56, Jan. 2000.

[33] T. P. White, R. C. McPhedran, C. M. de Sterke, L. C. Botten, and M. J. Steel, Confinement losses in microstructured optical fibers," Optics Letters, vol. 26, pp. 1660-1662, Nov. 2001.

[34] D. Ferrarini, L. Vincetti, M. Zoboli, A. Cucinotta, and S. Selleri, "Leakage properties of photonic crystal fibers," Optics Express, vol. 10, pp. 1314-1319, Nov. 2002.

[35] D. Ferrarini, L. Vincetti, M. Zoboli, A. Cucinotta, F. Poli, and S. Selleri, "Leakage losses in photonic crystal fibers," in Proc. Optical Fiber Communications Conference OFC 2003, Atlanta, Georgia, USA, Mar. 23-28, 2003,

[36] J. J. Refi, "Mixing True Wave TM RS Fiber with other Single-Mode Fibers in a network," Bell Laboratories Innovations, Lucent Technologies, Tech. Rep., 2001.

\section{Authors Biography}

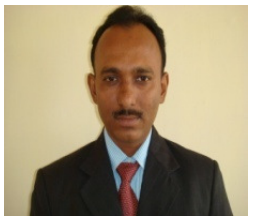

Mr. Sandhir K Singh received his M.Sc (Physics wirh specialization in Electronics) degree from Magadh University, Bodhgaya, Bihar in 1997. He is presently Research Scholar attached to PG Department of. Physics, VBU, Hazaribag. He is having more than 10 years of teaching experience. $\mathrm{He}$ is presently working in area of Photonic Crystal fiber and Optical Communication..

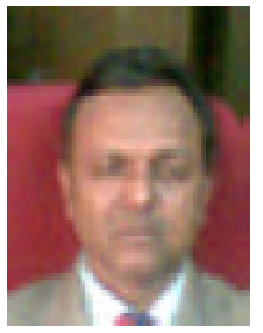

Dr. Dharmendra K Singh is presently working as Head, Department of Electronics and Communication \& Information Technology, BIT Sindri, Dhanbad. He has more than 20 years of teaching experience. He is heading the department of Electronics and Communication \& Information technology since 2002. He is instrumental in starting the curriculum on information technology. He has published more than 50 papers in journals and conferences. He has already supervised 01 thesis in computer Science \& Engg and 05 research scholars are presently enrolled for their doctoral degree. The area of research in which he works are Coding theory, cryptography, optical Amplifiers , Photonic Crystal Fibers, e-Governance and Educational Planning. He is member and conveners of various computerization programs of BIT Sindri, Vinoba Bhave University, Ranchi University, Dhanbad district and Jhakhand state. He is also member of various professional societies.

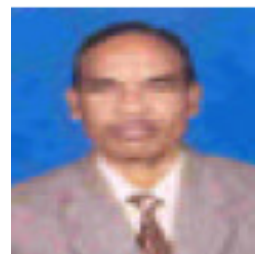

Dr. Parmanand Mahto did his M.Sc.(Physics) from Ranchi University and Ph.D in their field of X-ray Absorption Spectroscopy from Indian School of Mines, Dhanbad. Besides post doctoral work at IUC, Indore, he has got a number of research papers published in international journals of repute like Physical Review Letters, Solid State Communications, Indian Journal of physics etc. He has been a member of International XAFS Society, IAPT, ISCA etc. and has a teaching experience of 35 years at the undergraduate and postgraduate levels. His current research fields are XANES, EXAFS, Plasmons, Photonics, Lattice Distortion and Spintronics. 